\title{
HACIA UNA ARTICULACIÓN ENTRE TEORÍA DEL CAPITAL HUMANO Y LAS POLÍTICAS SOCIALES
}

\author{
Dante Boga \\ Universidad Nacional de Mar del Plata, Argentina
}

http://dx.doi.org/10.5209/NOMA.56427

\begin{abstract}
Resumen.- El presente trabajo pretende argumentar en torno a la existencia de un vínculo entre las políticas sociales focalizadas (políticas de transferencias condicionadas) y la teoría del capital humano. Para ello, se plantearán los elementos centrales de la teoría del capital humano y los debates que se desarrollan al respecto. Luego, se realizará un recorrido somero por la noción de política social y las transformaciones que se han desarrollado en esta materia a partir de las políticas de reforma estructural del Estado que se implementaron en Argentina y la región. Por otra parte, se plantearán los argumentos desarrollados por los organismos técnicos internaciones en torno a la noción de trasmisión intergeneracional de la pobreza ligada a la dotación de capital humano. Por último, la conclusión a la que se arriba es que la teoría del capital humano se constituye en un supuesto subyacente que estructura el funcionamiento de las políticas sociales focalizadas, específicamente en relación al componente de las condicionalidades.
\end{abstract}

Palabras clave: Estado - Políticas sociales- Capital humano- Focalización.

\section{Towards an articulation between the theory of human capital and social policies.}

Abstract.- This paper aims to argue about the existence of a link between focused social policies (conditioned transfer policies) and human capital theory. To this end, the central elements of the human capital theory and the debates that will be developed will be discussed. Then, a brief tour will be made for the notion of social policy and the transformations that have been developed in this matter from the policies of structural reform of the State that were implemented in Argentina and the region. On the other hand, the arguments developed by the international technical agencies on the notion of intergenerational transmission of poverty linked to the endowment of human capital will be raised. Finally, the conclusion is that the theory of human capital is an underlying supposed that structures the operation of targeted social policies, specifically in relation to the component of conditionalities.

Keywords: State - Social policies - Human capital - Targeting.
SUMARIO
1. Presupuestos teóricos acerca de la intervención social del estado.
2. Políticas sociales en Argentina
3. teoría del capital humano.
4. Políticas sociales, pobreza y capital humano.
5. Conclusiones
6. Bibliografía. 


\section{PRESUPUESTOS TEÓRICOS ACERCA DE LA INTERVENCIÓN SOCIAL DEL ESTADO.}

Las políticas sociales ha sido motivo de numerosas producciones teóricas y académicas en el contexto latinoamericano. Naturalmente esta tendencia tiene que ver con las particularidades de la región, en términos de las profundas desigualdades que la llevan a ser la región menos distributiva del planeta. Al mismo tiempo las conceptualizaciones están también imbricadas en la compleja trama de rupturas y continuidades en los modelos de protección social propios de cada realidad.

Dentro de las modalidades de intervención estatal el concepto de protección social resulta relevante para analizar. Este concepto pretende en la definición de Cecchini (et al, 2014) integrar el conjunto de medidas que los países adoptan para garantizar niveles de vida mínimos para la población. Es un instrumento para alcanzar las metas de bienestar físico y mental, educación y trabajo.

Específicamente para el análisis las políticas sociales, se requiere de una perspectiva amplia que sea capaz de explicar elementos centrales que la constituyen $e$ inciden en sus funcionamientos y determinaciones. En esta dirección el desarrollo de Adelantado et al (1998) se refiere a las relaciones entre política social y estructura social. Lo plantean como "(...) el abanico de interrelaciones entre la política social y la estructura social conduce hacia la idea de una sucesión de influencias bidireccionales con diversos tempos (...). Podría hablarse también, en otro lenguaje, de una cierta recursividad de la estructura social" (1998; 4).

Los autores dan cuenta de la complejidad existente a la hora de considerar las variables indispensables para analizar el fenómeno, de manera que postulan la interdependencia de esas variables'. "Las políticas sociales, serían, por tanto, uno de los factores primordiales que contribuyen al proceso de estructuración de las sociedades del capitalismo avanzado: surgen de la estructura social a la vez que contribuyen a configurar la misma." (Adelantado et al, 2000; 5).

Desde la perspectiva recursiva van a plantear que a la política social como moduladora y generadora de la desigualdad naturalizando e institucionalizando las desigualdades de clase, género, edad, etnia o cualquier otra.

La política social se articula en un conjunto de decisiones y actuaciones público-administrativas, generadas en la esfera estatal, que inciden directamente y de formas diversas sobre la organización y distribución de los recursos de bienestar, y lo hace mediante regulaciones, servicios

\footnotetext{
1 Los autores recurren a la teoría de la estructuración formulada por Anthony Giddens a principios de la década del 60, como critica al estructural-funcionalismo parsoniano que dio en denominar "consenso ortodoxo" (Cambiasso, 2011)
} 
y transferencias. La secuencia de tales decisiones a lo largo del tiempo da forma a la importancia relativa de las esferas, y articula los ejes de desigualdad al establecer las reglas sobre qué recursos se distribuyen, en qué proporción, a quién, en qué momento y de qué forma. Esa secuencia de decisiones está limitada por la capacidad de influencia de los actores en presencia, y limitada también por el contexto específico, que puede presentar restricciones de carácter estructural, estratégico, organizativo, político, de ciclo económico, demográfico $\mathrm{O}$ de cualquier otro tipo. (Adelantado et al, 1998; 18).

La política social es una mediación entre política y economía, es decir, entre el bienestar y el bien común; originalmente a partir de considerar un problema específico que era la cuestión obrera en Europa del siglo XIX. De manera que el Estado Social de las sociedades industrializadas asume y reconoce que requiere para superar fracturas sociales atender la satisfacción de necesidades de la población. En el siglo XXI la cuestión del "desarrollo humano integral". Se plantea entonces como "un dominio que se sitúa entre lo económico y lo político como medio de conservación o reforzamiento del poder el Estado" (De Laubier en Fernández Riquelme y Caravaca Llamas, 201 1; 5). Esto se completa con un sistema normativo e institucional que permite proveer, asistir y proteger.

Es posible plantear los siguientes "elementos generales de toda manifestación de la Política social los siguientes: generada en una época histórica concreta, determinada por una decisión política, realizada jurídicamente, con un estatuto científico concreto, e institucionalizada pluralmente." (Fernández Riquelme y Caravaca Llamas, 2011; 6).

Los autores diferencian dos cuestiones: la política social general y la política social específica. Por política social integral, entienden que se trata de una política de la sociedad fundada en "formas de intervención públicas en la vida social para resolver determinados problemas o cuestiones sociales (...) la forma política del estado social" (Molina en Fernández Riquelme y Caravaca Llamas, 2011; 13). En este sentido, se sustancian dos sentidos: uno material que pretende alcanzar el bienestar social y, el otro, formal que se plantea en término de derechos sociales. La política social específica propone satisfacer necesidades y derechos de los ciudadanos, aquí parecen los servicios asistenciales, los programas, etc.

En la perspectiva marxista la política social aparece como la "...la manera estatal de efectuar la transformación duradera de obreros no asalariados en obreros asalariados." (Offe, 1990; 3). En esta línea de interpretación se plantea que la política social viene a designar formas de existencia legítimas y legitimadas por el estado en los márgenes del trabajo. Se trata de intervención estatal de carácter normativo que regule y sancione políticamente quién es o no un asalariado, y organice 
formas de subsistencia legítimas, paralelas a la de intercambiar trabajo por salario. En similar línea de interpretación para Cortés y Marshall (1993) la política social refería a los instrumentos estatales de regulación de la fuerza de trabajo, las condiciones de vida y el conflicto social.

\section{POLITICAS SOCIALES EN ARGENTINA}

En Andrenacci y Soldano (2006) hay un minucioso recorrido de las teorías que fundamentan el estudio de las políticas sociales y de los cambios que han operado en los Estados Sociales. Particularmente plantean el problema de las transformaciones recientes en la cuestión social y la manera que la forma de protección estatal pasó de políticas universalistas que tenía como eje el empleo forma hacia políticas de lucha contra la pobreza. Este tipo de políticas comienzan de desarróllarse fuertemente a partir de la década del '90, específicamente a partir del conjunto de prescripciones que se operan desde el denominado Consenso de Washington. La noción básica de las políticas a partir de esto tuvo que ver principalmente con tres grandes preceptos: descentralización, focalización y participación (Grassi et al, 1994) ${ }^{2}$. Este periodo de reforma estructural del estado vino a cambiar la manera que la sociedad se vinculaba con la res pública. El Estado pasó a ubicarse en otro lugar de la trama social y de la trama política. Resulta relevante que este lugar no tiene que ver con lo que se ha planteado en términos de ausencia del estado, en realidad se trata de un proceso de reposicionamiento de las funciones del estado (Sassen, 2000) en donde emerge un nuevo orden y una nueva institucionalidad que se direcciona esencialmente hacia lo privado.

En Argentina la política social ha sido entendida históricamente a partir de tres grandes segmentos. Primero, el sistema de seguridad social que comprende al sistema previsional, a las obras sociales, a las pensiones, a los retiros por invalidez. Es decir, la protección social que se desprende del trabajo formal asalariado, un sistema de matriz indudablemente bismarckiana ${ }^{3}$. Segundo, las instituciones públicas universales por excelencia en el país: el sistema educativo público y el sistema público de salud. Por último, las intervenciones particulares dirigidas a sectores en situación de vulnerabilidad o que cumplían (tomando un léxico actual) los criterios de focalización. Según la interpretación de Isuani (2008) se trata de tres conceptos aplicables a cada uno de los segmentos: primero la contribución, segundo la ciudadanía y tercero la discrecionalidad)

\footnotetext{
2 Este tema será profundizado más adelante.

3 Resulta relevante considerar las distinciones que realiza Isuani (1991) entre el Estado de Bienestar (EB) de corte bismarckiano y las políticas económicas propias del Estado Keynesiano (EK). Para el autor: "...mientras el EK fue un período en el desarrollo del capitalismo que acabó minando la lógica de acumulación del capital, el EB lo antecedió, creció enormemente a su amparo y está en condiciones de sobrevivir sin él". (1991; 6)
} 
Esta suerte de convivencia de diferentes estrategias y tipos de políticas social se prorrogan hasta la actualidad. Para Lo Vuolo (1998) de trata de un "híbrido institucional" que combina estas tres formas claramente diferenciadas de intervención. A este modelo Filgueira lo ha caracterizado como en términos de ciudadanía estratificada, dado que el acceso a los derechos sociales va a estar vinculado al tipo de inserción socio ocupacional que cada actor posee. Sobre este asunto resulta relevante la noción ya expuesta de Adelantado (1998) en el sentido de la recursividad que genera la política social en torno a la estructura social. Del Valle (2012) recurre a la noción de "efecto Mateo" elaborada por Robert Merton para analizar este tema. El "efecto Mateo" permite "sostener que, en la estructura social se produce una acumulación diferencial de ventajas y desventajas por lo que "[...] los procesos de auto-selección individual y de selección social institucionalizada, interactúan y afectan las probabilidades sucesivas de acceso a la estructura de oportunidades [...]" (Merton en Del Valle, $2012 ; 101$

La década del '90 ha sido ampliamente analizada y caracterizada en diversos trabajos (García Delgado, 1994; García Delgado, 1998; Minujin, 1999). En ellos el Estado es caracterizado a partir de un apartamiento en sus funciones de regulación. Por otra parte, se plantea un modelo de sociedad volcado hacia el mercado como espacio donde resolver las necesidades sociales y dirimir los conflictos. El ajuste estructural vino a ser una manera de palear el golpe económico (Bernal-Meza, 1996) producido a fines de la década del '80. El mismo estaba caracterizado por altas tasas de inflación, desocupación y conflictividad social, en el contexto de la crisis de la deuda que padecían los países latinoamericanos (Teubal, 2005). La aceptación de las directrices emanadas de los organismos internaciones (como el Fondo Monetaria Internación o el Banco Mundial) así como las demandas tanto del Plan Brady como del consenso de Washington, configuraron un nuevo lugar para el Estado en la economía y una nueva concepción de la forma de intervención.

Bajo el proceso conocido como "Reforma del Estado" el gobierno promovió un conjunto de decretos que dieron lugar a la privatización de empresas estatales, la capitalización de créditos por parte de los acreedores del Estado, el despido de trabajadores como condición para las privatizaciones, la ejecución de los contratos de todo ente en que el Estado Nacional tuviera participación total o mayoritaria y la desregulación de las empresas privadas. En este escenario comienza a emerger una nueva cuestión social.

Al mismo tiempo se produjo una apertura de la economía y las políticas económicas se orientaron a conseguir la estabilidad de las cuentas externas y el saneamiento monetario. Para lograr este último objetivo, en 1991, Argentina impulsó un sistema de paridad cambiaria con el dólar conocido como Plan de Convertibilidad. 
Siguiendo la interpretación prevaleciente de los organismos internacionales que se basada en la teoría neoclásica, las reformas económicas puestas en marcha en la Argentina reforzarían al mercado como asignador de recursos y eliminarían aquellas distorsiones que afectaban negativamente la demanda laboral4.

En cuanto a la política social Grassi et al (1999) definió al modelo de intervención de Estado como neoliberalismo neoconservador, caracterizado particularmente por el asistencialismo. Es decir, la emergencia de una política social marcadamente focalizada en los sectores denominados excluidos de la sociedad. La exclusión social, en este modelo de sociedad, se plantea de una manera fragmentadora y estigmatizaste. Fragmentadora porque el tratamiento que se le dio a los sectores sociales excluidos, que en el paradigma hegemónico están constituidos por elementos disfuncionales. Es decir que donde hay una lógica que promueve y, es más, que requiere de la existencia de pobres y marginales, el imaginario simbólico creado en torno a la pobreza y la marginación, asume que se trata de la responsabilidad individual; problemas de adaptación, etc.

El funcionamiento es también estigmatizaste en las políticas sociales focalizadas. La focalización está en el eje de una forma de entender la sociedad, una sociedad dividida en estamentos, en segmentos 0 parcelas estancas entre las cuales no hay vínculo. La existencia de pobres tiene que ver con sus ingresos mensuales, la solución transferirles divisas para garantizar su subsistencia. Las políticas de transferencias condicionadas (PTC) son la forma tradicional de esta modalidad. Con diferentes bemoles esto es un patrimonio de la región latinoamericana, que con financiamiento compartido (habitualmente parte del financiamiento de estos programas proviene de organismos internaciones de crédito) han impulsado estos programas.

De esta manera la problemática de la pobreza y la vulnerabilidad social es atendida mediante los programas de transferencias condicionadas (PTC) se caracterizan por intentar combatirla mediante la combinación de un objetivo de corto plazo, el aumento de los recursos disponibles para el consumo a fin de satisfacer las necesidades básicas de las familias beneficiarias, con otro de largo plazo, el fortalecimiento del capital humano para evitar la transmisión intergeneracional de la pobreza (Britto, 2006; Cecchini y Madariaga, 2010; Cohen y Franco, 2006 ${ }^{a}$, León, 2008)5. Para ello, por lo general se utilizan tres instrumentos: i) las transferencias monetarias, para aumentar los ingresos, ii) el condicionamiento de las transferencias al uso de ciertos servicios sociales, para la acumulación de capital humano y iii) la focalización en

4 Del Valle (2001) se refiere, fundamentalmente, a los sesgos que afectarían negativamente las actividades de exportación, el sector agropecuario (urban bias) y el factor trabajo.

${ }_{5}^{5}$ Sobre este tema de regresará en el Capítulo III - Capital humano y políticas sociales. 
los hogares pobres y extremadamente pobres. Por medio de estos mecanismos, se intenta abordar de manera conjunta los aspectos materiales y cognitivos asociados a las situaciones de pobreza, asumiendo la importancia de combinar la protección social no contributiva con la promoción social (Levy y Rodríguez, 2005), así como de realizar las intervenciones sobre una base intersectorial (Cohen, Franco y Villatoro, 2006).

En la óptica de las políticas sociales se define a la focalización como la idea que descansa el supuesto de hacer más con menos y aumentar los aspectos progresivos del gasto social al dirigir los esfuerzos públicos a quienes más lo necesitan. Si bien en muchos casos las técnicas de focalización lograron dirigir los esfuerzos a la población propuesta, no siempre fue así; algunas veces trajeron consigo efectos indeseados. Ciertamente, la aplicación de estas nuevas modalidades de políticas sociales se prestó, en algunos casos, a la formación de clientelas porque, bajo la forma institucional que asumió en la región, se constituyó en instrumento altamente discrecional del Poder Ejecutivo. También creó lo que algunos analistas llaman "vigilantismo", el cual se registra cuando políticas e instrumentos de prueba de medios destruyen formas básicas de solidaridad entre los pobres, generando una distinción entre "pobres merecedores" y "no merecedores". Un riesgo adicional de la focalización es la quiebra de solidaridades entre clases y dentro de las mismas clases y la estigmatización de los destinatarios. (Filgueira et al, 2007).

\section{TEORÍA DEL CAPITAL HUMANO.}

Los estudios sobre capital humano se inician en el ámbito de la economía a mediados del siglo XX. La inquietud central de la teoría del capital humano a nivel macroeconómico es el análisis de los nexos entre los adelantos educacionales y el desarrollo económico de un país. A partir de esta idea se comienza a considerar el factor humano como determinante del desarrollo económico de una nación.

El concepto fue acuñado fundamentalmente por Gary Becker (1975) y Theodore Schultz (1961) ambos académicos de la Universidad de Chicago, sumamente influenciados por el pensamiento económico de Milton Friedman. La denominada Escuela de Chicago (de economía) va a definir el pensamiento económico a partir de la década del '70. La parte más sustancial y relevante de los planteos desarrollados van a discutir el keynesianismo a favor de monetarismo. El contexto en el que se planteó esto es la crisis mundial de los estados de bienestar, por un lado, y la influyente crisis generada a partir de 1973, llamada "Crisis del petróleo" (situación generada a partir de la decisión de los países miembros de la OPEP de modificar los precios del crudo y dejar de vender a los países que colaboraron con Israel en la Guerra de Yom Kipur). 
Como teoría económica es una suerte de reflujo de la teoría económica clásica (de ahí que asume como neoclásica) y va a funcionar como sustento teórico de los organismos internacionales de crédito $y$, específicamente para el caso de América Latina, una fundamentación de las medidas planteadas en el Consenso de Washington (Mujica Chirinos y Rincón Gonzalez, 2010).

Sin embargo, Falgueras (2008) ha rastreado que esta idea tiene precedentes clásicos como Adam Smith cuando introduce la noción de los beneficios que genera en el trabajador la especialización. De manera que "este concepto capta la idea de que las personas gastan en sí mismas parte de sus recursos más importantes (dinero y tiempo) de muy diversos modos" $(2008 ; 20)$ con el fin de mejorar la posición.

En marco de la teoría económica el capital humano es definido por Schultz y Becker como "la suma de las inversiones en educación, formación en el trabajo, emigración o salud que tienen como consecuencia un aumento en la productividad de los trabajadores" (Giménez, 2005; 104). Dentro de las consideraciones criticas acerca de la teoría del capital humano se argumentó las dificultades de calcular y conocer la relación entre la inversión inicial realizada y los ingresos futuros que devengan de la misma, resultando dificultoso aislar esa variable de otras (Calles, 1996).

Económicamente se enmarca en la teoría de la marginalidad. Desde la teoría neoclásica de la marginalidad se sostiene que a un aumento marginal de la escolaridad, correspondería un aumento marginal de la productividad. La renta es mantenida como función de productividad, donde, a una determinada productividad marginal, corresponde una renta marginal. En base a este pensamiento se infiere que la educación es un instrumento eficiente de distribución de la renta y equidad social.

Una de las principales limitaciones que tienen los estudios acerca del capital humano radica en que su utilización se lo vinculó casi exclusivamente, desde diferentes áreas de conocimiento, con la educación. Desde la economía específicamente en términos de inversión en educación y su posterior rendimiento. Villalobos y Pedroza (2009) han señalado las limitaciones del abordaje estrictamente económico de esta teoría y el escaso tratamiento y conceptualización que se ha realizado de la educación en este marco. Esto se plantea en términos de tasa de retorno, que se define como "la diferencia entre el producto y los salarios sobre los acervos netos del capital o alguna variable que indique la inversión realizada en un período de tiempo determinado" (Ruiz en Garrido Trejo, 2007; 5).

Sociológicamente, Gil Villa (1995) la inscribe a esta teoría en lo que denomina un funcionalismo tecno- económico que se vendría a nutrir de elementos de la sociología positiva de Saint-Simon, Comte y Durkheim, adhiriendo a una sociedad meritocrática en la que la 
educación formal, accesible a todos, sería el instrumento distribuidor clave.

La teoría viene a plantear un tipo racional de actor social que es capaz de evaluar permanentemente los costos y los beneficios de sus acciones (Gil Villa, 1995)6. De manera que sus elecciones estarán regidas por la optimización económica de las prácticas con el objetivo de acumular diferentes credenciales y conocimientos, lo cual le facilitará un espacio socio-ocupacional más redituable. Es decir, la educación es una inversión y como tal deberá Tuego generar beneficios, naturalmente estos beneficios deberán ser superiores a los costos originales que generó el acceso a los mismos; en tiempo, dinero, dispensa de otras actividades, etc. Esto se viene a encuadrar en una manera específica de entender la acción social que Weber plantea como "racional con arreglo a fines"; es decir, "el individuo valorara racionalmente las probables consecuencias de un determinado acto en los términos del cálculo de medios para un fin." (Guiddens, 1998; 253). En la elaboración previa de la teoría de la acción comunicativa elaborada por Habermas (1987) se plantea un concepto similar que denomina acción teleológica y viene a plantear que "el actor realiza un fin o hace que ser produzca el estado de cosas deseado eligiendo en una situación dada los medios más congruentes y aplicándolos de manera adecuada" (Habermas, $1987 ; 122){ }^{7}$

En los años sesenta, desde el paradigma del capital humano algunos autores notaron que las disparidades en las remuneraciones no se debían exclusivamente al factor educativo y consideraron otras variables (como el sexo, el entorno social, la educación de los padres, etc.) para complementar los estudios de las brechas de ingresos. (Van Raap, 2010). Deninson comienza a considerar la posibilidad de realizar un cálculo en torno a que porcentaje de los ingresos de las personas se podían vincular con el capital humano, entendiendo que había otras variables en juego. El autor suponía que el 60\% de los diferenciales de ingresos eran atribuibles a los efectos de la educación, a diferencia de Becker que planteaba que factores tales como las características socioeconómicas, el sexo, etc. explicaban sólo una pequeña parte de las diferencias de ingresos entre los trabajadores con título universitario y secundario (Morduchowicz, 2004).

En la tradición marxista se critica a esta teoría ya que asumen que es un intento más de la teoría económica neoclásica en eliminar la noción de clase social del análisis.

6 Gil Villa $(1995 ; 1)$ plantea objeciones al respecto de entender al estudiante como un actor racional "en busca de una inversión que rentabilice sus posibilidades sociales y económicas futuras"

7 La acción estrategia vienen a designar una ampliación de la acción teleológica e implica la noción de maximización de utilidad y expectativas de utilidad. 
Los atributos del trabajador, que son valorados por los empleados y que, por tanto, constituyen "capital humano", no se limitan a las cualificaciones técnicas y a las capacidades productivas abstractas. En concreto, los atributos de clasificación como la raza, el sexo, la edad, el origen étnico y las credenciales formales, considerados frecuentemente como irrelevantes dentro de la lógica de la producción capitalista, se usan para fragmentar a los trabajadores y reducir la formación potencial de coaliciones dentro de la empresa (Bowles y Gintis, 2014; 223)

Es posible cuestionar que la adquisición de herramientas, como por ejemplo las del sistema educativo, resulte un mecanismo igualador. Según Blaug (1983) el sistema educativo forma en un sector los "soldados de infantería" y en otro (especialmente la enseñanza superior) forma a los "tenientes y capitanes" de la economía. Según Bowles y Gintis (2014) se trata de una manera de control social para mantener la estabilidad del sistema capitalista, al tiempo que se genera que la estructura de oportunidades generada socialmente se asuma y se procese de forma individual: una subjetivación de los problemas sociales.

Un trabajo reciente elaborado por Vanina Van Raap (2010) ha trabajado en relación al tema de educación (pensando en clave de capital humano) y las políticas sociales vinculadas al acceso al mundo del trabajo. La hipótesis central de la autora sostiene que

"trayectorias laborales de los jóvenes se encuentran asociadas a factores estructurales que son propios de las características que asume el régimen social de acumulación de la Argentina, en donde opera de manera persistente una estructura socio-ocupacional segmentada que genera oportunidades diferenciales según posicionamientos sociales" (Van Raap, 2010; 15)

Para la autora el propósito que orientó su estudio era "demostrar que las políticas centradas en la capacitación laboral basadas en los supuestos del capital humano trasladan al plano individual problemáticas de carácter estructural." (Van Raap, 2010; 110).

Por último en este apartado, resulta relevante asumir una definición que resulte operativa para los fines del trabajo que se pretende desarrollar. En el análisis que efectúa Giménez (2005) para la CEPAL, se avanza en organizar algunos elementos de la teoría del capital humano, en principio, algunas cuestiones que quedan como autoevidentes (cosa sobre la que se regresará más adelante, en otro apartado). Por otra parte, existe en la literatura especializada homologaciones que no aportan a la comprensión: por ejemplo capital humano y educación formal; dejando por fuera un conjunto de elementos que resultan significativos. El autor propone un indicador acerca de la dotación de capital humano, en el cual se considera la educación tanto formal 
como informal y agrega que es necesario tener en cuenta la salud y la experiencia.

"Se considera que el capital humano puede tener un origen innato o adquirido. El capital humano innato comprende aptitudes de tipo físico e intelectual, que pueden verse modificadas debido a las condiciones de alimentación y salud. El capital humano adquirido se irá constituyendo a lo largo de la vida de los sujetos, a través de la educación formal, de la educación informal y de la experiencia acumulada. Estos tres tipos de formación adquirida van a condicionar la instrucción laboral y el sistema de valores de los sujetos, que determinarán, junto a las aptitudes innatas, su rendimiento en el trabajo." (Giménez, 2005; 106)

\section{POLITICAS SOCIALES, POBREZA Y CAPITAL HUMANO.}

En esta dirección, las políticas sociales que se comenzaron a diseñar a partir de fines de la década del ' 80 en Argentina y la región, comenzaron a portar tres criterios fundamentales: focalización, descentralización y participación8 (Grassi et al, 1994). Para las autoras esto fue un embate contra las políticas universalistas desarrolladas los últimos 40 años?. De manera que, como se verá más adelante, se comienza a plantear dentro de estas políticas cuestiones como la transferencias monetarias y la capacitación. La capacitación aparece como una manera de generar habilidades para poder competir en el mercado de trabajo de parte de los sectores vulnerables de la sociedad: la población objetivo.

Este criterio de "población objetivo" resulta central en esta construcción de la noción que el Estado llegue a aquellos que lo necesiten. Es decir que hay una supremacía de la noción de necesidad sobre la noción de ciudadanía social.

En el análisis de Adelantado y Scherer (2008) argumentan la debilidad de las políticas sociales focalizadas en torno a tres ejes: 1) debilitan la ciudadanía social, 2) favorecen el clientelismo político y, 3) son asistenciales, es decir que no constituyen un derecho. Para los autores las políticas sociales universales basadas en la ciudadanía social responden a una manera de procesar el conflicto social en un modelo de desarrollo que propone la industrialización por sustitución de importaciones. En este esquema tiene centralidad el trabajo: formal, masculino, jefe de familia. A partir de la década del 80 con el quiebre de este modelo se plantea las políticas sociales focalizadas como una manera viable de enfrentar la escasez fiscal. Estas son funcionales al sostenimiento al modelo neoliberal.

\footnotetext{
${ }^{9}$ Es necesario recordar que el texto fue elaborado hace más de veinte años.
} 
En el desarrollo de Ortiz Gómez (2014) al reflexionar sobre la construcción de un sujeto neoliberal, se plantea como la ética de la responsabilidad del Estado cede su lugar a una nueva ética que tiene que ver fundamentalmente con que los ciudadanos cubran los vacíos dejados, es decir, la responsabilidad individual frente a cuestiones producidas socialmente. En este sentido, los valores dominantes, o el sentido común neoliberal, asumen esto como un problema de tipo individual que se debe procesar y tratar de forma individual. Ortiz Gómez (2014) analiza esto en clave de modelo civilizatorio, retomando el planteo de Atilio Borón, que a partir de estas ideas intenta configurar una manera de organizar la vida social; es decir, el neoliberalismo como proyecto cultural.

Diferentes autores han caracterizado que la deficiente dotación de capital humano en los hogares redunda en un mecanismo de trasmisión intergeneracional de la pobreza (CEPAL 2006, Cohen y Franco, 2006 ; Britto, 2006; León, 2008; CEPAL, 2009; Cecchini y Madariaga, 2010; Kaztman, 2011; CEPAL 2014). Para el Programa de Naciones Unidas para el Desarrollo

"El capital humano de un país determina sus posibilidades de desarrollo y de construir una sociedad más igualitaria. La expansión del capital humano se basa en la evolución poblacional, en particular de la fuerza de trabajo complementada por flujos migratorios internacionales, y en niveles crecientes de salud y educación. Una sociedad más igualitaria requiere de un mayor capital humano, pero también de la expansión del derecho a la salud y la educación." (PNUD, 2013; 77)

En la CEPAL (2006 y 2009) se ha avanzado en considerar que las Políticas de Transferencias Condicionadas (PTC) tienen un objetivo a corto plazo que tiene que ver con la satisfacción de necesidades básicas mediante las transferencias monetarias, Por otra parte, se plantea que las condicionalidades (fundamentalmente salud y educación) tienen el propósito de ampliar la dotación de capital humano de los hogares.

"Los programas de transferencias condicionadas (PTC) representan una de las herramientas centrales en el marco de las políticas sociales de combate a la pobreza adoptadas por los gobiernos de la región. Son programas de carácter no contributivo que buscan aumentar los niveles de consumo de las familias por medio de transferencias monetarias -y así reducir la pobreza en el corto plazo- y fortalecer el capital humano de sus miembros para romper la reproducción intergeneracional de la pobreza. (...) La estratificación del capital humano de los hogares según el nivel socioeconómico está profusamente documentada y es el principal eslabón en la transmisión intergeneracional de la desigualdad, dado que el capital humano es el factor principal de las trayectorias laborales a lo largo de la vida productiva" (CEPAL, 2009; 30-33). 
En un informe posterior (CEPAL, 2014) vinculan la estructura productiva con desigualdad a través de las "capacidades productivas", es decir, mediante el capital humano. De hecho plantean que la desigualdad de "estas capacidades" (las desigualdades de capital humano) se traducen en desigualdades productivas y de remuneraciones. En este informe la comisión parece soslayar casi medio siglo de debate académico (al cual se ha remitido este trabajo sucintamente en el acápite anterior) e insiste en considerar la desigualdad como producto de las diferencias en la dotación de capital humano.

En el informe CEPAL (2015) se plantea la educación (en el mencionado informe no se menciona en ningún momento el concepto de capital humano) como una dimensión esencial para la igualdad, el bienestar de las personas y el desarrollo de los países. Se analiza en el informe el avance de la región en cuanto a la escolaridad, lo cual claramente es un dato auspicioso, pero que debe considerarse con bemoles cuando se lo correlaciona con la noción de igualdad.

Existen dos nociones que se oponen a este planteo y que entran en discusión abierta con la teoría del capital humano y la economía de la educación. Por un lado la categoría de ejercito industrial de reserva (Marx, 1973) y la noción de masa marginal ${ }^{10}$ (Nun, 1999)

La noción de ejército industrial de reserva o superpoblación relativa sugiere que el capitalismo requiere para su funcionamiento ciertos trabajadores que sean desocupados crónicos. Si la fuerza de trabajo es considerada una mercancía, nada impide que una gran divergencia entre su precio y su valor. Estos trabajadores actúan como un freno para los salarios de los trabajadores activos en su reclamo de salarios más altos. Es decir, en la teoría marxista se plantea que la clase capitalista pagará los salarios que mantengan a la clase obrera en un nivel de subsistencia.

Nun critica que Marx en su conceptualización engloba tres modalidades de superpoblación relativa diferentes: la latente, la estancada y la flotante. El autor plantea que la marginalidad se origina en el crecimiento control y monopolización del proceso de industrialización por parte del capital extranjero. El autor refiere que "la penetración de las corporaciones transnacionales en América Latina ha creado una sobrepoblación relativa y que parte de esta es afuncional o aun disfuncional para el capitalismo" (Kay, 1991; 8-9). Lo que viene a suceder es que esta superpoblación afuncional no juego el rol de ejército de reserva ya que nunca será absorbida por el sector productivo y no es capaz de disputar espacios dentro del aparato del mercado de trabajo, por lo tanto no tienen ninguna influencia en el salario de los trabajadores.

10 En similar línea de análisis Quijano (1977) propuso el concepto de mano de obra marginal y polo marginal de la economía. 
Del Valle (2009) realiza una crítica acerca de la teoría del capital humano partiendo de la concepción de la pobreza y la desigualdad como fenómenos dinámicos relacionados principalmente a la exclusión social. El trabajo enfatiza en lo inadecuado de estas líneas de análisis para resolver el problema de la pobreza. Para el autor hay una imposibilidad de asumir que la educación tiene que preparar a los pobres para el manejo y procesamiento de información como ha postulado la economía de la educación clásica. Esto claramente está ignorado las posiciones sociales y la distribución de la estructura de oportunidades (Van Raap, 2010).

Es posible pensar que "las medidas tendientes a seleccionar a los pobres y a activar su capital humano difícilmente sean efectivas, ya que se traducen en nuevos procesos que incrementan la competencia en un escenario de escasez de recursos estatales." (Del Valle, 2009; 229). En esta dirección, el autor concluye que las políticas de lucha contra la pobreza, específicamente las que tienen relación con la incorporación a alguna forma de inserción laboral precaria, "han hecho pasar a las personas de un estado de desocupación transitorio a una situación de transitoriedad permanente"

\section{CONSIDERACIONES FINALES.}

De acuerdo a su matriz de emergencia teórica, era esperable que esta categoría sea utilizada por un conjunto de actores que tiene que ver con el sector empresarial. La noción de capital humano y de recursos humanos está inscripta en la estructuración de grandes empresas como sectores específicos y relevantes. Analizar en sus dimensiones relevantes y su forma de instalación excede los objetivos de este trabajo y no es el interés que se persigue. Sin embargo, es un dato de interés considerar con está inscripta socialmente este categoría y la manera que ella se ha filtrado hacia otros sectores de una manera subrepticia y difícil de reconocer.

Como se analizado en el acápite anterior las políticas de desarrollo analizadas desde el Estado, así como las políticas sociales, en sus diferentes versiones y características llevan al capital humano como supuesto implícito o subyacente. En la literatura analizada se hace mención al capital humano como categoría y se destaca la necesidad de ampliarlo, fortalecerlo, generar dotación, etc. En todos los casos se asume como supuesto que estas capacidades y habilidades redundarán en modificar las situaciones de los sectores en situación de pobreza, desde un marcado sentido meritocrático. Se hacen todas estas consideraciones, pero no se realiza un análisis de que ha que se refiere el concepto de capital humano, mucho menos su historia, su marco político, ideológico y sociológico, sus debates teóricos no saldados, entre otras cuestiones. Se le da al concepto una entidad, un 
sentido autoevidente. Este problema es analizado en Bourdieu et al (2008) cuando plantea la idea de vigilancia epistemológica ${ }^{1}$.

La categoría se identifica con un ethos cultural que tiene que ver con las condiciones de producción del sistema capitalista en su conjunto y con el momento particular en el que se instala como principio ordenador de las políticas sociales focalizadas. La noción de la responsabilidad individual como eje que estructura las relaciones sociales.

Escriben Marx y Engels (1985) en "La ideología Alemana" acerca de la construcción y consolidación de las ideas dominantes.

"las ideas de la clase dominante son las ideas dominantes en cada época; o, dicho en otros términos, la clase que ejerce el poder materia dominante en la sociedad es, al mismo tiempo, su poder espiritual dominante. La clase que tiene a su disposición los medios para la producción material dispone con ello, al mismo tiempo, de los medios para la producción espiritual, lo que hace que se le sometan, al propio tiempo, por término medio, las ideas de quienes carecen de los medios necesarios para producir espiritualmente. Las ideas dominantes no son otra cosa que la expresión ideal de las relaciones materiales dominantes, las mismas relaciones materiales dominantes concebidas como ideas; por tanto, las relaciones que hacen de una determinada clase la clase dominante, o sea, las ideas de su dominación." (1985; 39)

En la posterior elaboración de Gramsci se plantea que las clases sociales, dominadas o subordinadas, participan de una concepción del mundo que les es impuesta por las clases dominantes (Gruppi, 1978). A esto lo denomina ideología de las clases, la cual es trasmite por diferentes canales: la escuela, la iglesia, el folclor, los medios de comunicación, el servicio militar.

"La hegemonía es esto: capacidad de unificar a través de la ideología y de mantener unido un bloque social que, sin embargo, no es homogéneo, sino marcado por profundas contradicciones de clase. Una clase es hegemónica, dirigente y dominante, mientras con su acción política, ideológica, cultural, logra mantener junto a sí un grupo de fuerzas heterogéneas e impide que la contradicción existente entre estas fuerzas estalle, produciendo una crisis en la ideología dominante y conduciendo a su rechazo, el que coincide con la crisis política de la fuerza que está en el poder" (Gruppi, 1978; 23).

11 "Confrontando continuamente a cada científico con una explicitación crítica de sus operaciones científicas y de los supuestos que implican y obligándolo por este medio a hacer de esta explicitación el acompañante obligado de su práctica y de la comunicación de sus descubrimientos, este sistema de controles cruzados tiende a constituir y reforzar sin cesar en cada uno la aptitud de vigilancia epistemológica." (Bourdiev et al, 2008:115) 
Introducir la noción de capital humano en el terreno de las políticas sociales implicar considerar como opera. En esta dirección, es posible plantearla como una superestructura ideológica que marca una concepción clara sobre el mundo social. Es decir, es una producción de la clase dominante para garantizar condiciones de dominio sobre los sujetos. Una subjetivación de los problemas sociales.

La lógica del neoliberalismo está en la línea de esta manera de considerar las problemáticas. Como explica Alemán (2014) el neoliberalismo "diferencia del liberalismo clásico o el neoconservadurismo, es una construcción positiva, que se apropia no sólo del orden del Estado, sino que es un permanente productor de reglas institucionales, jurídicas y normativas, que dan forma a un nuevo tipo de 'racionalidad' dominante" $(2014 ; 1)$. El autor plantea entonces el carácter constructivo del neoliberalismo y no solo su parte destructiva, en el sentido que procura producir un nuevo tipo de subjetividad. Este sujeto aparece como un emprendedor o inversor de sí (Gago, 2014) que busca la adaptación, el rendimiento, la competencia.

La noción de Gago (2014) acerca del sujeto inversor de sí es relevante para este estudio. Este sujeto es el que produce el neoliberalismo. Al decir de la autora: "...si se trata de pensar el neoliberalismo no sólo como una doctrina homogénea y compacta, es para poner el foco en la multiplicidad de niveles en los que opera, la variedad de mecanismos y saberes que implica y los modos en que se combina y articula, de manera desigual, con otros saberes y formas de hacer." (Gago, 2014; 18). Es decir, un neoliberalismo "desde abajo", inscripto y funcionando y diferentes instancias de la vida social, señalando el carácter poliformo que adquiere12. Para Gago la "...autoempresarialidad, la autogestión y, también, la responsabilidad sobre sí. Se trata de una racionalidad, además, no puramente abstracta ni macropolítica, sino puesta en juego por las subjetividades y las tácticas de la vida cotidiana." (2014; 10).

El capital humano puede entenderse también como una racionalidad propia del funcionamiento descripto en el marco neoliberal. Se trata de

12 El espacio concreto que analiza la autora en su investigación es: "un ensamblaje concreto que vincula a la feria La Salada con el taller textil clandestino y la villa (y la fiesta como forma de entrada y salida a cada una de esas situaciones). Se trata de una secuencia genealógica pero también revela una lógica de mutua contaminación, de permanentes reenvíos, de complementariedades y contradicciones. Nos interesan las trayectorias que se tejen entre la villa, el taller textil, la feria y la fiesta para mostrar los modos en que se entrometen una dentro de la otra. En la villa se renueva permanentemente la población migrante y es lugar de producción de una multiplicidad de situaciones laborales que van del autoemprendimiento, a la pequeña empresa pasando por el trabajo doméstico y comunitario, en relación de enrevesadas dependencias. Pero también en ella se 'sumerge' el taller textil clandestino para aprovecharla como espacio de recursos comunitarios, de protecciones y favores y de fuerza de trabajo." (Gago, 2014; 19) 
la noción moralizante desde el punto de vida de la responsabilidad individual.

Es posible establecer una relación entre las exigencias emanadas por la noción de capital humano y los principios ideológicos sobre los que se asienta el funcionamiento del sistema capitalista, desde el punto de vista de las ideas fuerza que le han dado sustento a lo largo de la historia. Para Weber el espíritu del capitalismo se funda en la lógica y cosmovisión que surge con el protestantismo. De manera que está caracterizado por una combinación de dedicación permanente por la ganancia de dinero de manera licita junto con una suerte de ascetismo que exime de cualquier derroche vinculado a los gustos personales. Una cultura del esfuerzo y de la austeridad que se introduce con un nuevo espíritu empresarial. El éxito personal estará guiado por esas conductas que resultan fundamentales. (Guiddens, 1998).

El advenimiento de las políticas sociales focalizadas o Políticas de Transferencias Condicionadas (PTC) trajo aparejado, se podría decir en la misma génesis, el estigma hacia las personas que los perciben. La focalización generó identificación y posteriormente la construcción de categorías sociales hacia quien es parte de la política. Las ideas fuerza que se fueron instalando tiene que ver con la que estas personas no tienen una iniciativa de trabajo, que son "vagos", etc. Impulsado por medios de comunicación e incluso por dirigentes de la política, estas ideas fomentan la construcción de un sentido común al respecto.

Por otra parte, el trabajo aparece planteado desde una moralidad que dignifica a aquel que participa. Al contrario de las categorías del marxismo que intentan visibilizar la explotación del capital sobre los trabajadores, el sentido común dominante le da al trabajo un valor en sí mismo, un espacio de dignidad y prevalencia.

Es importante en este punto considerar en qué lugar queda la noción de ciudadanía con la que se viene trabajando en este estudio. "El concepto de ciudadanía está íntimamente ligado, por un lado, a la idea de derechos individuales y, por el otro, a la noción de vínculo con una comunidad particular." (Kymlicka y Norman, 1997; 2). Para los autores es necesario ver la manera que la noción fue discutida la ciudadanía por diferentes sectores, pensando especialmente en el sentido de las discusiones entre izquierda y derecha. La ciudadanía social de Marshall fue presentada por las derechas como una forma de pasividad de los ciudadanos; inspirados en la idea de responsabilidad. Lo plantean en términos de "ciudadanía responsable" que claramente tiene antecedentes en la teoría económica clásica al dar un lugar central al mercado's. Una especie de desciudadanización a favor de

13 "Esta visión de la Nueva Derecha no quedó a salvo de objeciones. Por ejemplo, la afirmación de que el crecimiento de una subclase de desempleados dependientes de los programas de bienestar es un resultado de las propias políticas sociales desconoce el impacto de la reestructuración económica global, al tiempo que es difícilmente 
las relaciones de mercado en lugar de la relación entre los ciudadanos y el Estado.

Para los autores, los sectores de izquierda reafirman en argumento de Marshall a favor de que la condición de posibilidad para que alguien sea miembro pleno de una sociedad es que están satisfechas sus necesidades básicas, es decir, que se desarrolle por parte del Estado de manera concreto la ciudadanía social. En este punto se hace un énfasis especial en los derechos de participación precediendo a las responsabilidades.

Las condicionalidad en la política social vienen a pretender saldar al menos en parte esta discusión. De manera subrepticia la condicionalidad se plantea como una exigencia, como un hacer algo para recibir, una suerte de devolución o de merecimiento de eso. Socialmente resulta bastante claro que es necesaria una discusión acerca de las formas de existencias legítimas, es decir, por qué sería necesario merecer algo. En otras palabras, ¿cuál es el lugar de la ciudadanía a la hora de llevar adelante la condicionalidad? Y más allá todavía ¿̇se deja de ser ciudadano por no 'cumplir' con la condicionalidad? ¿Acaso la condicionalidad ciudadaniza a quien la realiza más que al que no la realiza?

Las políticas sociales focalizadas han sido diseñadas en el contexto que las sociedades redescubren la desigualdad (Andrenacci, 2003). En el contexto, como ya se ha analizado, que el modelos de desarrollo "hacia adentro" o que consideraba la industrialización como eje se había sido dado por terminado. Los países habían reiniciado su ciclo de endeudamiento y dependencia. Con la promesa de la modernización se cedieron los lugares centrales del Estado en la economía. Esto hizo surgir una población residual, sobre la cual había que intervenir de alguna manera desde el Estado. El diseño de las políticas sociales focalizadas consideró umbrales de subsistencia. No umbrales de dignidad de las personas. En otras palabras, los montos dispuestos no garantizan la vida digna de las personas que lo perciben, sino un mínimo, que necesariamente debe ser complementado con otro tipo de actividades. En un trabajo reciente se consideraba, desde esta línea argumental, que los ingresos de estas políticas pueden ser interpretados como una "subvención estatal a las empresas del sector informal" (Boga y Del Valle, 2015; 355).

Por último, considerar la noción de transitoriedad de las políticas sociales (Del Valle, 2009). Es decir, que las personas que están incluidas en ellas lo estarán un tiempo, ya que su situación es de anomalía en relación de las 'normales' relaciones productivas. La política social aquí despliega su potencial de capacitación para que la persona pueda disputar

compatible con el hecho de que algunos de los países en donde más se ha desarrollado el Estado de bienestar (en Escandinavia, por ejemplo) han registrado tradicionalmente las tasas más bajas de desempleo. (Kymlicka y Norman, 1997; 6) 
espacios en el medio laboral. Específicamente que pueda acceder a un trabajo formal que le permita acceder a la totalidad de la ciudadanía social.

Asumir este argumento es desconocer las condiciones de un mercado de trabajo en contracción, por un lado, y sumamente expulsivo, por el otro. En donde la capacidad para manejar información o técnicas, o la fuerza de trabajo y el tiempo de trabajo, incluso la voluntad de trabajo (es decir, la voluntad de acceder, por fin, a condiciones de explotación, pero que permiten garantizar las condiciones materiales de existencia) no son el criterio fundamental por el cual se puede o no acceder a un espacio socio-laboral. La problemática actual de la desocupación y de la precariedad laboral excede ampliamente las razones individuales. De manera que resulta de importancia visibilizar esta problemática. Dar cuenta y denunciar estos aspectos que se puede adjetivar como perversos del funcionamiento general de la política social focalizada. La responsabilización individual de temas y de problemas que son sociales. La subjetivación de la cuestión social, es quizás la mayor victoria que reálizó el neoliberalismo sobre la totalidad de la sociedad.

El capital humano aparece como una excusa de un sistema que en su conjunto no garantiza las posibilidades para que las personas accedan a condiciones dignas de existencia. Ciertamente, los niveles de exclusión son moralmente insostenibles y parafraseando a Offe es penoso pero importante afirmar que:

"...la exclusión no es un problema sino una situación con visos de fatalidad. La exclusión no es un problema porque la inclusión con garantías mínimas de derechos no es una solución realista y, por tanto, algo que pueda responsablemente fijarse como objetivo. De nada sirve cubrir esta situación dolorosa y fuera de control con capas de pomada todo lo gruesas que se quiera de la retórica socialdemócrata sobre la inclusión social. Tendremos que hacernos cargo a largo plazo de una situación en la que una gran parte de los miembros de nuestro mundo no va a encontrar acomodo en relaciones sociales y laborales 'normales'." (Offe, 1992; 52) 


\section{BIBLIOGRAFÍA.}

ADELANTADO, J; NOGUERA, J; RAMBLA Xavier, y SAEZ Luis (1998), Las relaciones entre estructura y políticas sociales: una propuesta teórica. En: Revista Mexicana de Sociología Número 3, Universidad Autónoma de México, México.

ADELANTADO, J y SCHERER, E (2008) Desigualdad, democracia, y políticas sociales focalizadas en América Latina. Revista chilena de gestión pública. Estado, gobierno y gestión pública, № 11 junio 2008.

ALEMÁN, Jorge (2014), Artículo: Neoliberalismo y Subjetividad. Diario Página 12, Contratapa. Buenos Aires.

ANDRENACCI, L (2003). Imparis Civitatis. Elementos para una teoría de la ciudadanía desde una perspectiva histórica. Sociohistórica, $n^{\circ}$ 13-14, 2003. ISSN 1852-160.

ANDRENACCl, L. y SOLDANO, D. (2006) Aproximación a las teorías de la política social a partir del caso argentino, en ANDRENACCI, LUCIANO Problemas de política social en la Argentina contemporánea, Buenos Aires, UNGS-Editorial Prometeo.

BECCARIA L. y LÓPEZ, N.(1996): El debilitamiento de los mecanismos de integración social en Beccaria, López, Sin Trabajo, UNICEF/LOSADA, Buenos Aires.

BECKER, G. (1975). El Capital Humano. Alianza Universidad Textos, Madrid.

BERNAL-MEZA, R. (1996) ¿La globalización, un proceso y una ideología? Revista realidad latinoamericana, N 139, abril-mayo 1996.

BLAUG, M. (1983): "El status empírico de la teoría del capital humano: una panorámica ligeramente desilusionada" en Toharia, L.: El mercado de trabajo: Teorías y aplicaciones, Editorial Alianza, Madrid.

BRITTO, T.F. (2006) 'Conditional transfers in Latin America', Poverty Focus, June.

BOGA, D. y DEL VALLE, A. (2015) Protección Social y Transformaciones de las Políticas Sociales en Argentina. Revista Textos \& Contextos (Porto Alegre), v. 14, n. 2, p. 338 - 358, ago./dez. 2015

BORDIEU, P.; CHAMBOREDON, J. y PASSERON, J. (2008) El oficio de sociólogo

, Buenos Aires, Siglo XXI

BOWLES, S y GINTIS, H (2014). "El problema de la teoría del capital humano: una crítica marxista." Revista de Economía Crítica, n¹8, segundo semestre 2014, ISNN 2013-5254

CAMBIASSO, Mariela (2011). La teoría de la estructuración de Anthony Giddens: un ensayo crítico. VI Jornadas de Jóvenes Investigadores. 
Instituto de Investigaciones Gino Germani, Facultad de Ciencias Sociales, Universidad de Buenos Aires, Buenos Aires

CALLES, M (1996). "Crítica a la teoría del capital humano". V JORNADAS DE ECONOMIA CRITICA SANTIAGO DE COMPOSTELA 17 Y 18 DE MAYO DE 1996.

CECCHINI, S y MADARIAGA, A. (2011) Programas de Transferencias Condicionadas. Balance de la experiencia reciente en América Latina y el Caribe, Cuadernos de la Cepal No. 95. Santiago de Chile: CEPAL.

CECCHINI, S; ROBLES, C Y FILGUEIRA, F. (2014) Sistemas de protección social en América Latina y el Caribe. Una perspectiva comparada. CEPAL, Chile.

CECCHINI, S. Y MARTÍNEZ, R. (2011) "Protección social inclusiva en América Latina. Una mirada integral, un enfoque de derechos". Santiago de Chile, Comisión Económica para América Latina y el Caribe (CEPAL).

CEPAL (2006). La protección social de cara al futuro: Acceso, financiamiento y solidaridad. Naciones Unidas. Santiago de Chile.

CEPAL (2009) Panorama Social de América Latina 2009. Naciones Unidas, Santiago de Chile.

CEPAL (2014) Panorama Social de América Latina 2014. Naciones Unidas, Santiago de Chile.

CEPAL (2015) Panorama Social de América Latina 2014. Naciones Unidas, Santiago de Chile.

COHEN, E. Y FRANCO, R. (coords.) (2006) Transferencias con Corresponsabilidad: Una mirada latinoamericana. México: Facultad Latinoamericana de Ciencias Sociales.

COHEN, E. ROLANDO, F. y VILLATORO, P. (2006) . "México: El programa de Desarrollo Humano Oportunidades" en Transferencias con corresponsabilidad. Una mirada latinoamericana. México. Secretaría de Desarrollo Social. (SEDESOL)

CORTÉS, R. y MARSHALL, A. (1993) Política social y regulación de la fuerza de trabajo. en Cuadernos Médico-Sociales n 65-66; Rosario.

DEL VALLE, A. (2001) Estrategias y Prácticas Sindicales: la reforma laboral en Argentina, Univ. De Barcelona, Barcelona. TESEO-UNICEF. Microfilm

DEL VALLE, A. (2009) Educación y pobreza la hipótesis del capital individual y el capital social. Co-herencia, vol.6, Núm.10, enerojunio,2009, pp 207-237. Universidad Eafit, Colombia.

DEL VALLE, A. (2012) Protección social, acción estatal y estructura de riesgos sociales. Textos \& Contextos (Porto Alegre), v. 11, n. 1, p. 52 - 64, jan./jul. 2012 
DRAIBE, S. Y RIESCO, M. (2006). Estado de bienestar, desarrollo económico y ciudadanía: algunas lecciones de la literatura contemporánea. México D.F. Comisión Económica para América Latina y el Caribe (CEPAL).

FALGUERAS, I. (2008) "El capital humano en la teoría económica: Orígenes y evolución" en Temas actuales de economía. Capital Humano. Instituto de análisis económico y empresarial de Andalucía. Volumen $\mathrm{N}^{\circ} 2,2008$.

FILGUEIRA, F. (2007) "Cohesión, riesgo y arquitectura de protección social en América Latina". Santiago de Chile, Comisión Económica para América Latina y el Caribe (CEPAL).

GAGO, V. (2014), "La razón neoliberal. Economías barrocas y pragmática popular". Editorial Tinta Limón, colección Nociones Comunes. Buenos Aires.

GARCÍA DELGADO, D. (1994). Estado y sociedad: la nueva relación a partir del cambio Estructural. Grupo Editorial Norma. Buenos Aires.

GARCÍA DELGADO, D. (1998). Estado-nación y globalización. Grupo Editorial Planeta, Ariel. Buenos Aires.

GARCIA DELGADO D. y NOSETTO L., (2004). La Ciudadanía en una Etapa de Reconstrucción: Imaginarios y Desafíos. Boletín TOP, Boletín virtual.

GARRIDO TREJO, C. (2007). "La educación desde la teoría del capital humano y el otro". Educere. Artículos arbitrados. ISSN: 1316 - 4910 - Año 11, N³6 • Enero - Febrero - Marzo 2007.

GASPARINI, L. y CRUCES, G. (2010) "Las asignaciones universales por hijo. Impacto, discusión y alternativas". Documento de Trabajo CEDLAS No 102, UNLP, La Plata.

GIL VILLA, F (1995) "El estudiante como actor racional: objeciones a la teoría del capital humano" Revista de Educación, núm. 306 (1995), págs. 315.327.

GIMÉNEZ, G (2005). La dotación de capital humano de América Latina y el Caribe. Revista de la CEPAL 8 6- Agosto 2005.

GRASSI, E.; HINTZE, S. y NEUFELD, M. (1994). Políticas Sociales, crisis y ajuste estructural. Espacio Editorial. Buenos Aires.

GRUPPI, L (1978). El concepto de hegemonía en Gramsci. México: Ediciones de Cultura Popular)

GUIDDENS, A. (1998). El capitalismo y la moderna teoría social. Idea Books SA. Barcelona.

HABERMAS J. (1987). Teoría de la acción comunicativa I, Taurus, Madrid.

ISUANI, E. (2008) La política social argentina en perspectiva. En Los programas sociales en Argentina hacia el Bicentenario: visiones y 
perspectivas /compilado por Guillermo Cruces... [et.al.]. - la ed. Buenos Aires: Banco Mundial.

ISUANI, E (1991). Bismarck o Keynes: ¿quién es el culpable? En ISUANI, Ernesto; LO VUOLO, Rubén y TENTI FANFANI, Emilio: El Estado Benefactor. Un paradigma en crisis; Buenos Aires, Miño y Dávila/CIEPP, 1991.

KAY, C. (1991). Teorías latinoamericanas del desarrollo. Nueva Sociedad. No 113, mayo-junio 1991.

KAZTMAN, R. (2011) Infancia en América Latina: Privaciones habitacionales y desarrollo de capital humano. Comisión Económica para América Latina y el Caribe (CEPAL). Naciones Unidas, Santiago de Chile.

KYMLICKA W. y NORMAN, W., 1997. El retorno del ciudadano. Una revisión de la producción reciente en teoría de la ciudadanía, en Ágora, No 7, pp. 5-42.

LEÓN, A. (2008) Progresos en la reducción de la pobreza extrema en América Latina. Dimensiones y políticas para el análisis de la primera meta del Milenio. Proyecto CEPAL -

AECID. Seguimiento del componente de pobreza del primer objetivo de desarrollo del Milenio (AEC/06/003).19

LEVY, S. y RODRÍGUEZ, E. (2005) Sin herencia de pobreza. El programa Progresa -Oportunidades de México. BID- Planeta. Ciudad de México D. F.

LO VUOLO, R. (1998) ¿Una nueva oscuridad? Estado de Bienestar, crisis de integración social y democracia"; en BARBEITO, A. y LO VUOLO, R. "La nueva oscuridad de la política social. Del Estado populista al neoconservador". Buenos Aires-Madrid, CIEPP-Miño y Dávila.

MARX, K. (1973) EL CAPITAL, México, FCE, 1973; tomo 1.

MARX, K. Y ENGELS, F. (1985). La Ideología Alemana. Buenos Aires: Ediciones Pueblos Unidos y Editorial Cartago.

MINUJíN, A. (1999). ¿La gran exclusión? Vulnerabilidad y exclusión social en América Latina. EUDEBA. Buenos Aires.

Ministerio de Salud de la Nación -MSA- (2008) Dirección de estadística y consulta en salud, CONSULTA EN LÍNEA. http://www.deis.gov.ar/

Ministerio de Trabajo, Empleo y Seguridad Social (2009) "Impacto de la Asignación Universal por Hijo en la pobreza y el ingreso de los hogares (estimación al $2^{\circ}$ trimestre de 2009)", Buenos Aires.

MORDUCHOWICZ, A (2004): "Discusiones en economía de la Educación" Editorial Losada. Buenos Aires, IIPE.

MUJICA CHIRINOS, N. y RINCÓN GONZALEZ, S. (2010) El concepto de desarrollo: posiciones teóricas más relevantes. Revista Venezolana de Gerencia, Año 15 № 50. 
NUN, J (1999) El futuro del empleo y la tesis de la masa marginal. Instituto de Desarrollo Económico y Social. Disponible en ttp://www.jstor.org/stable/3467265

OFFE, C. (1976) La política social y la teoría del Estado (1990). En libro: Economía Social Contradicciones en el Estado de Bienestar. Editorial Alianza, Madrid.

OFFE, K. (1992). La Sociedad del Trabajo. Madrid: Alianza.

ORTIZ GÓMEZ M.G., 2014. El perfit del ciudadano neoliberal: la ciudadanía de la autogestión neoliberal, en Sociológica, vol.29 № 83, México.

PNUD (2013). Informe Nacional sobre Desarrollo Humano 2013. Argentina en un mundo incierto: asegurar el desarrollo humano del siglo XXI. Naciones Unidas.

QUIJANO, A. (1977) Notas sobre el concepto de marginalidad social. Santiago de Chile. CEPAL

SASSEN, S. (2000) Nueva geografía política. Un nuevo campo transfronterizo para actores públicos y privado. Texto de la conferencia del Millenium.

SCHULTZ, T. (1961a) "Inversión en capital humano", en BLAUG, M. (1968) Economía de la Educación. Ed. Tecnos, Madrid, 1972, pp.15-32.

TEUBAL, M (2005). Reflexiones sobre la deuda. Revista Argumentos. UBA. Buenos Aires.

VAN RAAP, V. (2010) Tesis de maestría: Educación, políticas sociales y acceso al mundo del trabajo: un estudio acerca de la desigualdad de oportunidades para los jóvenes en la Argentina. Disponible en ceyds.sociales.uba.ar/files/2014/06/t8_10.pdf

VILLALOBOS MONROY, G. y PEDROZA FLORES, R. (2009) "Perspectiva de la teoría del capital humano acerca de la relación entre educación y desarrollo económico." Tiempo de Educar, vol. 10, núm. 20, juliodiciembre, 2009, pp. 273-306. Universidad Autónoma del Estado de México. Toluca, México 